\title{
Reabilitação pulmonar em longo prazo na doença pulmonar obstrutiva crônica
}

\section{Pulmonary rehabilitation in chronic obstructive pulmonary disease}

Cintia Laura Pereira de Araujo ${ }^{1,2,3}$, Manuela Karloh ${ }^{1,4}$, Karoliny dos Santos $^{1,3}$, Cardine Martins dos Reis ${ }^{1,3}$, Anamaria Fleig Mayer ${ }^{1,3,5}$

${ }^{1}$ Núcleo de Assistência, Ensino e Pesquisa em Reabilitação Pulmonar do Centro de Ciências da Saúde e do Esporte da Universidade do Estado de Santa Catarina (UDESC) Florianópolis (SC), Brasil.

${ }^{2}$ Curso de Especialização em Fisioterapia Cardiorrespiratória da Faculdade Inspirar (FI) - Curitiba (PR), Brasil.

${ }^{3}$ Programa de Pós-Graduação em Fisioterapia do Centro de Ciências da Saúde e do Esporte da UDESC - Florianópolis (SC), Brasil.

${ }^{4}$ Programa de Pós-Graduação em Ciências Médicas da Universidade Federal de Santa Catarina (UFSC) - Florianópolis (SC), Brasil.

${ }^{5}$ Departamento de Fisioterapia do Centro de Ciências da Saúde e do Esporte da UDESC - Florianópolis (SC), Brasil.

DOI: http://dx.doi.org/10.7322/abcshs.v39i1.246

\section{RESUMO}

Introdução: A doença pulmonar obstrutiva crônica (DPOC) prejudica o estado funcional, com consequente limitação das atividades de vida diária (AVD). Este estudo teve como objetivo investigar o efeito de um programa de reabilitação pulmonar (RP), em longo prazo, no estado funcional, na dispneia e no índice BODE em pacientes com DPOC. Relato de caso: Trata-se de um estudo retrospectivo e documental, com análise dos prontuários de cinco pacientes participantes de um programa de RP por um ano. Destes prontuários foram coletados dados referentes às avaliações: espirometria, índice de massa corporal (IMC), escalas London Chest Activity of Daily Living (LCADL) e Medical Research Council (MRC) e teste de caminhada de seis minutos (TC6min). Após um ano participando do programa de exercício físico, a maioria dos pacientes apresentou maior capacidade funcional, menor dispneia e redução no risco de mortalidade. Conclusão: Um ano de RP parece ter função de manutenção da melhora da capacidade funcional de pacientes com DPOC obtida após 24 sessões de treinamento.

Palavras-chave: doença pulmonar obstrutiva crônica; exercício; tolerância ao exercício; atividades cotidianas; mortalidade.

\begin{abstract}
Introduction: The chronic obstructive pulmonary disease (COPD) impairs the functional status with consequent impairment of the activities of daily living (ADL). This study aimed to investigate the effect of a long term pulmonary rehabilitation (PR) on the functional status, dyspnea and on the BODE index in patients with COPD. Case report: This is a retrospective and descriptive study. The records of five patients that participated in a year PR program were analyzed. Assessments about spirometry, body mass index (BMI), London Chest Activity of Daily Living (LCADL) and Medical Research Council (MRC) scales and six-minute walking test (6MWT) were collected. After a year participating in the PR program, most patients improved their functional capacity, dyspnea and reduced the risk of mortality. Conclusion: A year of PR seemed to maintain the improvement in functional capacity obtained after 24 training sessions in patients with COPD.
\end{abstract}

Keywords: pulmonary disease, chronic obstructive; exercise; exercise tolerance; activities of daily living; mortality. 


\section{INTRODUÇÃO}

A doença pulmonar obstrutiva crônica (DPOC) caracteriza-se por alterações pulmonares e importante comprometimento sistêmico $^{1,2}$. Sua progressão leva ao surgimento e piora da dispneia, perda de força muscular periférica e limitação na realização das atividades de vida diária $(\mathrm{AVD})^{1,2}$, o que está associado a um maior número de exacerbações e maior risco de mortalidade ${ }^{3}$.

Apesar da ineficácia da maioria dos tratamentos no declínio da função pulmonar ${ }^{1,4}$, estudos demonstram que programas de reabilitação pulmonar (RP) baseados em exercício físico reduzem a dispneia e aumentam a capacidade de exercício ${ }^{2}$, os quais são considerados preditores independentes de mortalidade, com o índice de massa corporal (IMC) e o grau de obstrução ao fluxo aéreo ${ }^{5}$. Sabe-se que programas de RP mais longos produzem efeitos mais duradouros. Após 12 semanas de RP, a capacidade funcional parece atingir um platô em pacientes com DPOC ${ }^{2}$. Embora os efeitos da RP já tenham sido bem documentados, a maioria foi determinada a partir de programas com protocolos de treinamento de seis a 12 semanas $^{6}$.

Este estudo teve como objetivo verificar os efeitos de um programa de RP, em longo prazo, no estado funcional, na dispneia e no risco de mortalidade em pacientes com DPOC.

\section{RELATO DE CASO}

Trata-se de um relato de caso retrospectivo, elaborado por meio da revisão de 11 prontuários de pacientes com DPOC, atendidos durante o período de março de 2009 a junho de 2011 na Clínica Escola de Fisioterapia do Centro de Ciências da Saúde e do Esporte da Universidade do Estado de Santa Catarina (CEFID/UDESC).

Foram incluídos os sujeitos que participaram do programa de RP da Clínica Escola de Fisioterapia do CEFID/UDESC por um período igual ou superior a um ano. Os participantes que por algum motivo não realizaram determinado componente do programa, possuíam frequência inferior a três vezes por semana, não eram assíduos ou não assinaram o Termo de Consentimento Livre e Esclarecido foram excluídos do estudo.

Dos prontuários foram extraídos dados espirométricos como: percentual do previsto da capacidade vital forçada
(CVF\%prev), percentual do previsto do volume expiratório forçado no primeiro segundo ( $\mathrm{VEF}_{1} \%$ prev) e a relação $\mathrm{VEF}_{1} / \mathrm{CVF}$ (pós-broncodilatador); dados antropométricos (peso, altura e IMC); distância percorrida no Teste de Caminhada de Seis Minutos (TC6min) - valores previstos segundo a equação de Iwama et al. ${ }^{7}$, índice $\mathrm{BODE}^{5}$ e escores das escalas de AVD London Chest Activity of Daily Living $(\text { LCADL })^{8}$ e dispneia da Medical Research Council (MRC) ${ }^{9}$. Tais variáveis foram coletadas em três momentos distintos: na avaliação inicial, após 24 sessões e após um ano de participação no programa.

A RP era realizada três vezes por semana e com duração de uma hora e meia, conforme recomendado pela American Thoracic Society/European Respiratory Society (ATS/ERS) ${ }^{2}$ e pelo American College of Chest Physicians/American Association of Cardiovascular and Pulmonary Rehabilitation (ACCP/AACVPR) ${ }^{6}$. O programa de exercício físico consistiu em treino aeróbio em esteira ergométrica durante $40 \mathrm{~min}$, a $60 \%$ da velocidade média do TC6min. A progressão da carga ocorria de acordo com a sensação de dispneia na escala modificada de Borg, a qual deveria estar entre quatro e seis. Além de treino aeróbio, realizou-se treino localizado de membros superiores (focando resistência muscular, com número alto de repetições, a 50\% de $1 \mathrm{RM}$ e exercícios de diagonais de facilitação neuromuscular proprioceptiva) e inferiores (fortalecimento de quadríceps e tríceps sural, duas a três séries de seis a 12 repetições com carga de trabalho entre $50-85 \%$ de $1 \mathrm{RM})^{2}$. Os pacientes também receberam orientações sobre cuidados na DPOC. Os profissionais responsáveis pela supervisão do treinamento eram fisioterapeutas. Todos os pacientes tinham acompanhamento médico (pneumologista) frequente e psicológico quando necessário.

Os dados coletados são apresentados em valores individuais. O estudo foi aprovado pelo Comitê de Ética em Pesquisa Envolvendo Seres Humanos da UDESC (141/2011).

Dos 11 prontuários revisados, cinco foram incluídos. Dos excluídos, dois foram por frequência inferior a três vezes por semana, um por assiduidade insuficiente, um por participação inferior a um ano, um por medicação não otimizada no período e outro por não realização de treinamento aeróbio. Os pacientes incluídos

Tabela 1: Caracterização dos sujeitos

\begin{tabular}{|c|c|c|c|c|c|c|c|c|}
\hline Paciente & Sexo & Idade (anos) & IMC $\left(\mathbf{k g} / \mathbf{m}^{2}\right)$ & $\begin{array}{l}\text { Tabagismo } \\
\text { (anos/maço) }\end{array}$ & $\begin{array}{l}\text { VEF }_{1} \\
\% \text { prev }\end{array}$ & $\begin{array}{l}\text { CVF } \\
\text { \%prev }\end{array}$ & VEF $_{1} / C V F$ & GOLD \\
\hline 1 & M & 59 & 25 & 44 & 40 & 68 & 47 & 3 \\
\hline 2 & M & 58 & 25 & 69 & 25 & 53 & 38 & 4 \\
\hline 3 & M & 66 & 31 & 132 & 32 & 38 & 64 & 3 \\
\hline 4 & M & 54 & 25 & 80 & 37 & 62 & 47 & 3 \\
\hline 5 & M & 60 & 22 & 114 & 23 & 51 & 36 & 4 \\
\hline
\end{tabular}

IMC: índice de massa corporal. VEF \% prev: percentual do previsto do volume expiratório forçado no primeiro segundo; CVF\%prev: percentual do previsto da capacidade vital forçada; VEF,/CVF: relação VEF,/CVF; GOLD: classificação da gravidade da doença pulmonar obstrutiva crônica segundo GOLD 
Tabela 2: Teste de caminhada de seis minutos

\begin{tabular}{|c|c|c|c|c|c|c|c|c|}
\hline \multirow{2}{*}{ Paciente } & \multicolumn{3}{|c|}{ Distância (m) } & \multirow{2}{*}{$\begin{array}{c}\Delta \text { Absoluta } \\
\text { (m) }\end{array}$} & \multirow{2}{*}{$\begin{array}{c}\Delta \text { Percentual } \\
(\%)\end{array}$} & \multicolumn{3}{|c|}{ \%Prev } \\
\hline & Pré & $24^{a}$ & 1 ano & & & Pré & $24^{a}$ & 1 ano \\
\hline 1 & $267^{*}$ & 448 & 391 & 124 & 47 & 46 & 78 & 68 \\
\hline 2 & 414 & 494 & 364 & -50 & -12 & 72 & 86 & 63 \\
\hline 3 & 415 & 434 & 414 & -1 & $-0,2$ & 74 & 77 & 74 \\
\hline 4 & 425 & 520 & 538 & 113 & 27 & 73 & 89 & 92 \\
\hline 5 & 363 & 475 & 469 & 106 & 29 & 63 & 83 & 82 \\
\hline
\end{tabular}

*teste interrompido por dispneia e/ou dessaturação. Distância: distância em metros no Teste de Caminhada de Seis Minutos; $\triangle$ Absoluta: variação em metros entre a avaliação inicial e após um ano; $\triangle$ Percentual: variação em percentual da distância percorrida na primeira avaliação e após um ano; \%Prev: percentual percorrido em relação à distância prevista para o paciente; Pré: avaliação inicial; 24a: avaliação após a 24aㅗ sessão; 1 ano: avaliação após um ano

tinham idade entre 54 e 66 anos, três pertenciam ao estádio III (grave) e dois ao estádio IV (muito grave) da doença, de acordo com GOLD ${ }^{1}$ (Tabela 1).

$\mathrm{Na}$ avaliação inicial, o paciente um, que percorreu a menor distância no TC6min, apresentou dessaturação induzida pelo exercício (Tabela 2). O mesmo paciente realizava o treino aeróbio com suporte de oxigênio a $3 \mathrm{~L} / \mathrm{min}$. Após 24 sessões, quatro participantes alcançaram melhora clinicamente significativa da distância percorrida no TC6min, com aumento superior a $25 \mathrm{~m}^{10}$, e, após um ano de treinamento, quatro mantiveram essa melhora em relação à avaliação inicial (Figura 1). Os pacientes quatro e cinco apresentaram distância percorrida superior a $80 \%$ do previsto $^{7}$ após um ano de participação no programa, considerada, na prática clínica, capacidade de exercício normal.

$\mathrm{Na}$ escala LCADL, apenas o paciente um obteve aumento no escore após um ano de reabilitação, o que está relacionado à piora na limitação das AVD (Tabela 3). Em relação aos escores da LCADL e MRC, o paciente cinco apresentou, inicialmente, o maior grau de limitação em AVD na avaliação inicial. Entretanto, este obteve a melhora mais relevante em ambas as escalas após um ano de treinamento físico.

Após um ano de RP, o paciente quatro passou para um quartil de maior risco de mortalidade, o que se justifica pela redução do $\mathrm{VEF}_{1} \%$ prev. O paciente dois também apresentou piora do $\mathrm{VEF}_{1} \%$ prev; entretanto, esta foi compensada pela diminuição do grau de dispneia na escala MRC no cálculo do índice de mortalidade (Tabela 4).

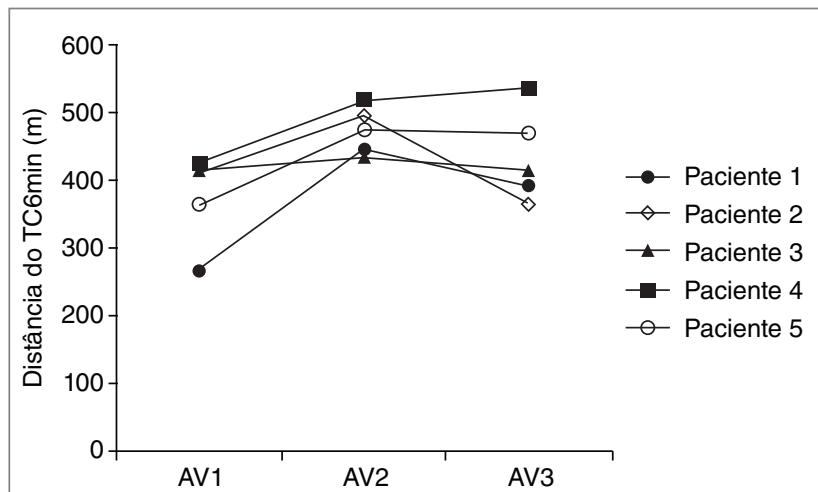

Distância TC6min: distância percorrida em metros no Teste de Caminhada de Seis Minutos; AV1: avaliação pré-reabilitação pulmonar; AV2: avaliação após a $24^{a}$ sessão; AV3: avaliação após um ano

Figura 1: Desempenho no Teste de Caminhada de Seis Minutos ao longo de um ano de reabilitação pulmonar

Tabela 3: Escala London Chest Activity of Daily Living

\begin{tabular}{|c|c|c|c|c|c|c|}
\hline \multirow{2}{*}{ Paciente } & \multicolumn{3}{|c|}{ Total } & \multicolumn{3}{c|}{ Total (\%) } \\
\hline 1 & Pré & $\mathbf{2 4}^{\mathbf{a}}$ & $\mathbf{1}$ ano & Pré & $\mathbf{2 4}^{\mathbf{a}}$ & $\mathbf{1}$ ano \\
\hline 2 & 27 & 31 & 32 & 36 & 44 & 43 \\
\hline 3 & 26 & 20 & 14 & 52 & 44 & 31 \\
\hline 4 & 20 & 18 & 14 & 40 & 36 & 28 \\
\hline 5 & 29 & 12 & 18 & 43 & 20 & 30 \\
\hline
\end{tabular}

Total: escore absoluto; Total (\%): escore percentual do total da escala London Chest Activity of Daily Living; Pré: avaliação inicial; 24a: avaliação após a $24^{a}$ sessão; 1 ano: avaliação após um ano

Tabela 4: Índice BODE

\begin{tabular}{|c|c|c|c|c|c|c|c|c|c|c|c|c|}
\hline \multirow{2}{*}{ Paciente } & \multicolumn{2}{|c|}{ VEF $_{1} \%$ prev } & \multicolumn{2}{|c|}{ IMC $\left(\mathrm{kg} / \mathrm{m}^{2}\right)$} & \multicolumn{2}{|c|}{ MRC } & \multicolumn{2}{|c|}{ TC6min (m) } & \multicolumn{2}{|c|}{ BODE pontuação } & \multicolumn{2}{|c|}{ BODE quartil } \\
\hline & Pré & 1 ano & Pré & 1 ano & Pré & 1 ano & Pré & 1 ano & Pré & 1 ano & Pré & 1 ano \\
\hline 1 & 40 & 41 & 25 & 26 & 3 & 3 & 267 & 391 & 5 & 4 & 3 & 2 \\
\hline 2 & 25 & 19 & 25 & 25 & 3 & 2 & 414 & 364 & 5 & 4 & 3 & 2 \\
\hline 3 & 32 & 32 & 31 & 32 & 2 & 2 & 415 & 414 & 3 & 3 & 2 & 2 \\
\hline 4 & 37 & 25 & 25 & 25 & 3 & 3 & 425 & 538 & 4 & 5 & 2 & 3 \\
\hline 5 & 23 & 26 & 22 & 22 & 4 & 1 & 363 & 469 & 6 & 4 & 3 & 2 \\
\hline
\end{tabular}

VEF,\%prev: percentual do previsto do volume expiratório forçado no primeiro segundo; IMC: índice de massa corporal; MRC: escala Medical Research Council; TC6min: distância percorrida no Teste de Caminhada de Seis Minutos 


\section{DISCUSSÃO}

Este relato de caso teve como objetivo levantar informações sobre o efeito, em longo prazo, de um programa de RP no estado funcional, na dispneia e no índice BODE em pacientes com DPOC. Sabe-se que o exercício físico não é capaz de alterar a função pulmonar; contudo, a redução da sensação de dispneia e o aumento da capacidade de exercício são benefícios inerentes à RP em pacientes com DPOC ${ }^{4}$.

Um dos instrumentos de avaliação utilizados para medir o estado funcional foi o TC6min, teste que tem alto poder prognóstico, sendo capaz de refletir a capacidade de exercício, as limitações nas AVD, além de ser sensível às mudanças proporcionadas pela $\mathrm{RP}^{11}$. Neste relato de caso verificou-se que, após realizarem 24 sessões, cinco pacientes aumentaram a distância percorrida no TC6min em no mínimo $25 \mathrm{~m}$, obtendo, portanto, uma melhora clinicamente importante ${ }^{10}$. Todavia, quando comparada aos resultados após um ano de reabilitação pulmonar, notou-se que a distância percorrida, após as 24 sessões, foi superior à distância alcançada após um ano em quatro dos cinco pacientes. Em relação à limitação em AVD, avaliada pela escala LCADL, uma redução acima de 5,91 pontos no escore total, valor médio de responsividade, indica melhora do paciente ${ }^{12}$. Quatro pacientes apresentaram redução igual ou superior a 5,91 pontos ${ }^{12}$. Apenas o paciente um obteve aumento no escore após um ano de reabilitação. Entretanto, é importante salientar a necessidade de oxigênio no treinamento aeróbio durante esse período de treinamento, já que este paciente, na sua avaliação, apresentou dessaturação aos esforços. Quanto ao percentual do total da LCADL, Simon et al. ${ }^{13}$ demonstraram que um escore superior a $50 \%$ indica grave limitação nas AVD. Neste relato de caso, os pacientes dois e cinco apresentaram, inicialmente, limitação grave. Após 24 sessões e um ano de treinamento, nenhum paciente mostrou esse nível de limitação.

Outra forma de determinar a repercussão da dispneia na DPOC é pela escala MRC, um dos componentes do índice
BODE, com o TC6min, $\mathrm{VEF}_{1}$ e IMC, o qual pode ser utilizado para avaliar o efeito de programas de $\mathrm{RP}^{14}$. No presente estudo, quatro pacientes tiveram melhora desse índice, passando para um quartil inferior. A redução do risco de mortalidade relacionou-se ao aumento da distância percorrida no TC6min em um paciente e, nos outros três, à redução da dispneia na escala MRC e à melhora do $\mathrm{VEF}_{1} \%$ prev. em um paciente. Cote e Celli ${ }^{14}$ observaram melhora de 19\% nesse índice após oito semanas de RP, porém, após dois anos de cessação do programa, os valores se igualaram aos do início da reabilitação. Em estudo de Foglio et al. ${ }^{15}$ também foi encontrada melhora desse índice após oito a dez semanas de reabilitação, mas houve piora ao longo de sete anos de programa, causada pela diminuição da distância percorrida do TC6min e aumento do escore da escala MRC, provavelmente relacionado à progressão da doença, por fatores como piora da função pulmonar, da limitação por troca gasosa e ventilatória, da disfunção musculoesquelética e estado inflamatório.

No presente estudo, o aumento do risco de mortalidade em um paciente, após um ano, esteve relacionado ao agravamento da função pulmonar. Cote e Celli ${ }^{14}$ verificaram que pacientes que não participam de programas de reabilitação pulmonar têm aumento do risco de mortalidade de $4 \%$ após um ano e $18 \%$ após dois anos. Esse aumento após dois anos foi associado ao declínio do $\mathrm{VEF}_{1}$ (20 $\mathrm{ml}$ no grupo que participou da reabilitação e $160 \mathrm{ml}$ no grupo não participante), diminuição da distância percorrida no TC6min (14 m e 53 m, respectivamente) e aumento da dispneia (diminuição de 0,14 pontos no escore da escala MRC no grupo que participou da reabilitação e aumento de 0,38 pontos no grupo não participante).

Diante dos dados encontrados neste relato, verifica-se que a maioria dos pacientes com DPOC apresentou melhora do estado funcional, redução da dispneia e do risco de mortalidade após 24 sessões de RP. Entretanto, após um ano de RP, a magnitude destes benefícios não aumentou, possivelmente alcançando apenas a manutenção destes.

\section{REFERÊNCIAS}

1. Vestbo J, Hurd SS, Agusti AG, Jones PW, Vogelmeier C, Anzueto A, et al. Global strategy for the diagnosis, management, and prevention of chronic obstructive pulmonary disease: GOLD executive summary. Am J Respir Crit Care Med. 2013;187(4):347-65. http://dx.doi.org/10.1164/rccm.201204-0596PP

2. Spruit MA, Singh SJ, Garvey C, Zuwallack R, Nici L, Rochester C, et al. An official american thoracic society/european respiratory society statement: key concepts and advances in pulmonary rehabilitation. Am J Respir Crit Care Med. 2013;188(8):e13-64. http://dx.doi.org/10.1164/rccm.201309-1634ST

3. Garcia-Aymerich J, Lange P, Benet M, Schnohr P, Anto JM. Regular physical activity reduces hospital admission and mortality in chronic obstructive pulmonary disease: a population based cohort study. Thorax. 2006;61(9):772-8.

http://dx.doi.org/10.1136/thx.2006.060145

4. Troosters T, Gosselink R, Decramer M. Short- and long-term effects of outpatient rehabilitation in patients with chronic obstructive pulmonary disease: a randomized trial. Am J Med. 2000;109(3):207-12. http://dx.doi.org/10.1016/S0002-9343(00)00472-1

5. Celli BR, Cote CG, Marin JM, Casanova C, Montes de Oca M, Mendez RA, et al. The body-mass index, airflow obstruction, dyspnea, and exercise capacity index in chronic obstructive pulmonary disease. N Engl J Med. 2004;350(10):1005-12. http://dx.doi.org/10.1056/NEJMoa021322 
6. Ries AL, Bauldoff GS, Carlin BW, Casaburi R, Emery CF, Mahler DA, et al. Pulmonary Rehabilitation: Joint ACCP/AACVPR Evidence-Based Clinical practice guidelines. Chest. 2007;131(5 Suppl):4S-42S.

http://dx.doi.org/10.1378/chest.06-2418

7. Iwama AM, Andrade GN, Shima P, Tanni SE, Godoy I, Dourado VZ. The six-minute walk test and body weight-walk distance product in healthy Brazilian subjects. Braz $\mathrm{J}$ Med Biol Res. 2009;42(11):1080-5 http://dx.doi.org/10.1590/S0100-879X2009005000032

8. Carpes MF, Mayer AF, Simon KM, Jardim JR, Garrod R. The Brazilian Portuguese version of the London Chest Activity of Daily Living scale for use in patients with chronic obstructive pulmonary disease. J Bras Pneumol. 2008; 34(3):143-51.

http://dx.doi.org/10.1590/S1806-37132008000300004

9. Kovelis D, Segretti NO, Probst VS, Lareau SC, Brunetto AF, Pitta F. Validation of the Modified Pulmonary Functional Status and Dyspnea Questionnaire and the Medical Research Council scale for use in Brazilian patients with chronic obstructive pulmonary disease. J Bras Pneumol. 2008;34(12):1008-18. http://dx.doi.org/10.1590/\$1806-37132008001200005

10. Holland AE, Hill CJ, Rasekaba $T$, Lee A, Naughton MT, McDonald CF. Updating the minimal important difference for six-minute walk distance in patients with chronic obstructive pulmonary disease. Arch Phys Med Rehabil. 2010;91(2):221-5. http://dx.doi.org/10.1016/j.apmr.2009.10.017

11. ATS Committee on Proficiency Standards for Clinical Pulmonary Function Laboratories. ATS statement: guidelines for the sixminute walk test. Am J Respir Crit Care Med. 2002;166(1):111-7. http://dx.doi.org/10.1164/ajrccm.166.1.at1102

12. Garrod R, Paul EA, Wedzicha JA. An evaluation of the reliability and sensitivity of the London Chest Activity of Daily Living Scale (LCADL). Respir Med. 2002;96(9):725-30. http://dx.doi.org/10.1053/rmed.2002.1338

13. Simon KM, Carpes MF, Correa KS, dos Santos K, Karloh M Mayer AF. Relationship between daily living activities (ADL) limitation and the BODE index in patients with chronic obstructive pulmonary disease. Rev Bras Fisioter. 2011;15(3):212-8.

14. Cote CG, Celli BR. Pulmonary rehabilitation and the BODE index in COPD. Eur Respir J. 2005;26(4):630-6. http://dx.doi.org/10.1183/09031936.05.00045505

15. Foglio K, Bianchi L, Bruletti G, Porta R, Vitacca M, Balbi B, et al. Sevenyear time course of lung function, symptoms, health-related quality of life, and exercise tolerance in COPD patients undergoing pulmonary rehabilitation programs. Respir Med. 2007;101(9):1961-70. http://dx.doi.org/10.1016/j.rmed.2007.04.007 